\title{
SPECTRAL RADIUS AND HAMILTONICITY OF GRAPHS
}

\author{
GUIDONG YU ${ }^{1,2}$, Yi FANG ${ }^{1}$, \\ Yizheng FAn $^{3}$ And Gaixiang CAi ${ }^{1}$ \\ 1 School of Mathematics \& Computation Sciences \\ Anqing Normal University \\ Anqing 246133, China \\ 2 Basic Department, Hefei Preschool Education College \\ Hefei 230013, P.R. China \\ 3 School of Mathematical Sciences \\ Anhui University \\ Hefei 230039, China \\ e-mail: guidongy@163.com \\ 1041098329@qq.com \\ caigaixiang@sina.com \\ fanyz@ahu.edu.cn
}

\begin{abstract}
In this paper, we study the Hamiltonicity of graphs with large minimum degree. Firstly, we present some conditions for a simple graph to be Hamilton-connected and traceable from every vertex in terms of the spectral radius of the graph or its complement, respectively. Secondly, we give the conditions for a nearly balanced bipartite graph to be traceable in terms of spectral radius, signless Laplacian spectral radius of the graph or its quasicomplement, respectively.
\end{abstract}

Keywords: spectral radius, singless Laplacian spectral radius, traceable, Hamiltonian-connected, traceable from every vertex, minimum degree.

2010 Mathematics Subject Classification: 05C50, 05C45, 05C35.

\section{REFERENCES}

[1] J.A. Bondy and V. Chvátal, A method in graph theory, Discrete Math. 15 (1976) 111-135. doi:10.1016/0012-365X(76)90078-9 
[2] J.A. Bondy and U.S. Murty, Graph Theory (Springer, New York, 2008).

[3] I. Tomescu, On Hamiltonian-connected regular graphs, J. Graph Theory 7 (1983) 429-436. doi:10.1002/jgt.3190070407

[4] B.L. Li and B. Ning, Spectral analogues of Erdös' and Moon-Moser's theorems on Hamilton cycles, Linear Multilinear Algebra 64 (2016) 2252-2269. doi:10.1080/03081087.2016.1151854

[5] B.L. Li and B. Ning, Spectral analogues of Moon-Moser's theorem on Hamilton paths in bipartite graphs, Linear Algebra Appl. 515 (2017) 180-195. doi:10.1016/j.laa.2016.11.024 The 15,1(2018).

[6] A. Bhattacharya, S. Friedland and U.N. Peled, On the first eigenvalue of bipartite graphs, Electron. J. Combin. 15 (2008) \#R144.

[7] M. Fiedler and V. Nikiforov, Spectral radius and Hamiltonicity of graphs, Linear Algebra Appl. 432 (2010) 2170-2173. doi:10.1016/j.laa.2009.01.005

[8] L.H Feng and G.H Yu, On three conjectures involving the signless Laplacian spectral radius of graphs, Publ. Inst. Math. (Beograd) (N.S.) 85 (2009) 35-38. doi:10.2298/PIM0999035F

[9] M. Ferrara, M. Jacobson and J. Powell, Characterizing degree-sum maximal nonhamiltonian bipartite graphs, Discrete Math. 312 (2012) 459-461. doi:10.1016/j.disc.2011.08.029

[10] Y.Z. Fan and and G.D. Yu, Spectral condition for a graph to be Hamiltonian with respect to normalized Laplacian, (2012).

[11] Y. Hong, J. Shu and K. Fang, A sharp upper bound of the spectral radius of graphs, J. Combin. Theory Ser. B 81 (2001) 177-183. doi: $10.1006 /$ jctb.2000.1997

[12] M. Lu, H.Q. Liu and F. Tian, Spectral radius and Hamiltonion graphs, Linear Algebra Appl. 437 (2012) 1670-1674. doi:10.1016/j.laa.2012.05.021

[13] R. Li, Egienvalues, Laplacian eigenvalues and some Hamiltonian properties of graphs, Util. Math. 88 (2012) 247-257.

[14] R. Li, Spectral conditions for some stable properties of graphs, J. Combin. Math. Combin. Comput. 88 (2014) 199-205.

[15] R. Liu, W.C. Shiu and J. Xue, Sufficient spectral conditions on Hamiltonian and traceable graphs, Linear Algebra Appl. 467 (2015) 254-266. doi:10.1016/j.laa.2014.11.017

[16] B. Ning and J. Ge, Spectral radius and Hamiltonian properties of graphs, Linear Multilinear Algebra 63 (2015) 1520-1530. doi:10.1080/03081087.2014.947984 
[17] B. Ning and B. Li, Spectral radius and traceability of connected claw-free graphs, Filomat 30 (2016) 2445-2452. doi:10.2298/FIL1609445N

[18] V. Nikiforov, Some inequalities for the largest eigenvalue of a graph, Combin. Probab. Comput. 11 (2002) 179-189. doi:10.1017/S0963548301004928

[19] V. Nikiforov, Spectral radius and Hamiltonicity of graphs with large minimum degree, Czechoslovak Math. J. 66 (2016) 925-940.

doi:10.1007/s10587-016-0301-y

[20] O. Ore, Note on Hamilton circuits, Amer. Math. Monthly 67 (1960) 55. doi:/10.2307/2308928

[21] G.D. Yu and Y.Z. Fan, Spectral conditions for a graph to be Hamilton-connected, Appl. Mechanics and Materials 336-338 (2013) 2329-2334. doi:10.4028/www.scientific.net/AMM.336-338.2329

[22] G.D. Yu, M.-L. Ye, G.-X. Cai and J.-D. Cao, Signless Laplacian spectral conditions for Hamiltonicity of graphs, J. Appl. Math. 2014 (2014) ID 282053. doi:10.1155/2014/282053

[23] G.D. Yu, Spectral radius and Hamiltonicity of a graph, Math. Appl. 27 (2014) 588-595.

[24] G.D. Yu, R. Li and B.H. Xing, Spectral invariants and some stable properties of a graph, Ars Combin. 121 (2015) 33-46.

[25] B. Zhou, Signless Laplacian spectral radius and Hamiltonicity, Linear Algebra Appl. 432 (2010) 566-570. doi:10.1016/j.laa.2009.09.004

Received 22 September 2017

Revised 10 January 2018

Accepted 10 January 2018 